\title{
Indumentaria masculina en la corte de Castilla a mediados del siglo XV: prendas de ir desnudo en la Cámara Real de Juan II el último año de su reinado
}

\author{
Laura Vegas Sobrino \\ Departamento de Historia del Arte. Universidad de Valladolid. \\ Junta de Castilla y León. Fondo Social Europeo. \\ lauravegassobrino@hotmail.com
}

\begin{abstract}
RESUMEN
A partir de la relación entre fuentes documentales y gráficas, se ofrece una descripción de algunas de las prendas que componían el traje masculino en la corte de Juan II, sus materiales, partes y adornos. Estas prendas permiten en ocasiones reconstruir el traje utilizado en determinadas ceremonias por el Rey, así como los trajes de corte de sus músicos, donceles, etc., y junto al mobiliario textil con el que conforman una unidad, conocer la estética de los ambientes generados en la corte para actos ceremoniales. Dentro de la Historia del Traje, constituyen una fuente de primer orden para el estudio de la indumentaria castellana en el periodo gótico tardío, ya que en este momento es el traje masculino de corte el que marca las pautas estéticas de la moda, y lo hace a partir del entorno cortesano presidido por el Rey, para lo cual las fuentes utilizadas aportan datos de primera mano.
\end{abstract}

Palabras clave: Juan II de Castilla; prendas; siglo XV; tejidos indumentaria; vestido.

\section{Male Castilian court clothing in the Mid-Fifteenth Century: some garments in the House-hold of John II}

\begin{abstract}
We provide a detailed description of some of the fabrics that made up the male suit in the retinue of John II, materials, parts and trim. These garments and accessories make sometimes possible rebuild the costume used by the King, his musicians, pages, etc., in court ceremonies, and get to know the aesthetic atmosphere in ceremonial cutting. The sources used provide first hand information for History of Costume, and they are a prime source for Spanish clothing studies from late Gothic period, as in this moment the court men's dress set fashion.
\end{abstract}

Key words: Fifteenth Century; garment; John II of Castile; Medieval dress; textile materials. 
La principal dificultad en el estudio de la indumentaria castellana de mediados del siglo XV es las escasez de prendas conservadas, y frente a otras cortes contemporáneas, de documentación y representaciones minuciosas.

Este trabajo constituye una aportación formal en aspectos referentes a la nomenclatura, materiales, proceso de confección, y partes de algunas de las prendas de la Cámara de Juan II. Para ello, en primer lugar se han recopilado los datos publicados en estudios previos sobre Castilla y Aragón, y luego se ha llevado a cabo una interrelación entre los datos de cargos, tajos y datas en dicha Cámara en $1453^{1}$, y fuentes gráficas contemporáneas, en especial el manuscrito de la Anacephaleosis de Alfonso de Cartagena de la Biblioteca Real de Palacio, fechada hacia $1460^{2}$, en el que se representa a Reyes y miembros de la corte. No es por tanto un estudio holista, pero sí riguroso en las descripciones y definiciones que, utilizando terminología específica y siguiendo la organización documental de la época, divide las prendas atendiendo a sus materiales y el lugar que ocupan respecto a otras en el conjunto del traje.

El documento analizado es una fuente de primera mano para conocer el guardarropa de Juan II, si bien es cierto que como es el único de este tipo del que se tiene constancia hasta el momento, la interpretación de sus datos permite sólo una visión parcial de los procesos de confección, conservación y uso de las prendas, así como de los oficios vinculados a la Cámara, y de las prendas y objetos que conformaban su conjunto. Sin embargo, sirve para poner en paralelo a la corte castellana con otras como la de Saboya o Borgoña, para las que gracias a la conservación de los registros de sus respectivas Cámaras de los paños en años sucesivos, se ha podido conformar ya una idea completa respecto al vestido de la corte ${ }^{3}$.

La metodología de análisis seguida consiste en relacionar, siguiendo las distintas fases del proceso de confección, principalmente el corte de paños y la data de prendas, los materiales exteriores e interiores de cada prenda, calculando cuando es posible la suma del coste de todos ellos (los precios de hechura no aparecen, al tratarse de profesionales de la propia Cámara), y por último, asociando cada prenda con el personaje al que se destina. Cuando ha sido posible, se ha puesto en relación cada prenda con otras prendas y objetos con los que formaría parte de un mismo traje.

1 Archivo General de Simancas (AGS), sección Casa y Sitios Reales (C y SR), legajo 42, folio 1 b-h. Vid.: CAÑAS GÁLVEZ, Francisco de Paula, "La música en la Corte de Juan II de Castilla (1406-1454). Nuevas fuentes para su estudio", en Revista de Musicología, XXIII (2000), n² 2, pp. 367-394.

2 CARTAGENA, Alonso de, Anacephaleosis, Biblioteca Real de Palacio (BRP), Madrid, manuscrito II / 3009. Este manuscrito ya fue utilizado como fuente por Carmen Bernis en BERNIS, Carmen, Trajes y modas en la España de los Reyes Católicos. II Los hombres, Madrid, Instituto Diego Velázquez del Consejo Superior de Investigaciones Científicas, 1979, figs. 10, 23 y 24.

3 Vid.: PAGE, Agnès, Vêtir le prince: Tissus et couleurs à la cour de Savoie (1427-1447), Fondation Humbert II et Marie José de Savoie, Cahiers Lausannois d'Histoire Médiévale, 8. Université de Lausanne, Lausanne, 1993; y JOLIVET-JACQUET, Sophie, "Pour soi vêtir honnêtement à la cour de monseigneur le duc de Bourgogne»: costume et dispositif vestimentaire à la cour de Philippe le Bon de 1430 à 1455. Tesis doctoral bajo la dirección de Vincent Tabbagh, Universidad de Borgoña, 2003. 


\section{PRENDAS DE IR DESNUDO}

Las prendas "de ir desnudo", calzas y jubón, constituían el armazón de la silueta masculina, de hombros anchos, con el vientre marcado por una ligera curva convexa paralela a la cóncava del final de la espalda, y piernas sutiles y estilizadas, a lo que contribuía un calzado terminado en punta.

Ambas tenían un patrón ceñido y estaban enforradas y guarnecidas ${ }^{4}$ para ajustarse, por lo que resultarían estrechas y de consistencia rígida. Cubrían la totalidad del cuerpo desde el cuello a los tobillos, uniéndose en la cintura con cintas de cuero con puntas rígidas, que además podían aparecer en otros lugares, con los que sujetar a ellas otras prendas textiles o metálicas. Se llevaban sobre las "prendas interiores" (bragas y camisa), y bajo las prendas "de vestir en cuerpo"5 (ropas y sayas).

Resulta revelador que en el manuscrito de la Anacephaleosis, dedicado a la genealogía de los reyes castellanos, no se represente ninguna figura "desnuda", es decir, todos los jubones aparecen cubiertos bien por corazas, bien por sayas o ropas de diferentes largos y hechuras. Esto hace pensar que aunque ir en jubón fuese aceptable, y el concepto de "ir desnudo" no fuese equiparable al de hoy en día, no se contemplaba para figuras nobles ni de cierta dignidad dentro de la corte.

Por otra parte, en el documento utilizado para este estudio no aparecen cortadas ni dadas prendas interiores como camisas o bragas. Sí hay constancia de que este tipo de prenda fuese utilizado por el Rey porque se menciona una taleguilla que el monarca portaría entre el camisón y el jubón. Puede que la Cámara contase con un número de ellas suficiente, cortadas con anterioridad, por lo que no aparecen en ninguno de los registros, si bien es cierto que sí se mencionan numerosos lienzos, que era el tipo de tejido utilizado para su confección.

\section{LAS CALZAS}

Las calzas podían ir desde el muslo (con altura variable) hasta los tobillos, pero desde mediados del siglo aparecen las braguetas ${ }^{6}$, necesarias para vestir las calzas con ropas cortas dando total cobertura a ingles y glúteos. La existencia de una abertura en la costura delantera de unión (la bragueta) hace pensar en la existencia de un tiro, es decir, aunque se habla de "pares" de calzas, éstas constituirían en el caso mencionado una prenda única. Contaban además con al menos otra costura que iría desde el talón por el centro de la pierna hasta la espalda. Su largo sobre el pie también era variable: algunas llevan avampiés o cubrición del empeine, y otras lo dejaban al aire, o incluso incorporaban suelas. Los estudios previos recogen noticias de muchos tipos de calzas

4 "Guarnecer" es aplicar una tela o piel distinta a la de la prenda, contrastando su textura o color, como refuerzo o para dotar de mayor empaque o comodidad.

5 La expresión vestir en cuerpo es propia de la época. BERNIS, Carmen, (1979), op.cit., p. 14, «La expresión vestir a cuerpo, usada en nuestros días, existía en forma casi idéntica en el siglo XV [...] en cuerpo».

6 BERNIS, Carmen, "El traje masculino en Castilla durante el último cuarto del siglo XV", en Boletín de la Sociedad española de excursiones, 54, 1950, pp. 191-236 (p.200). 
de las que se desconocen sus características específicas, aunque es posible que algunas se nombraran por el lugar de procedencia del tejido de confección.

Entre abril y diciembre de 1453, en la Cámara se confeccionaron un total de 37 pares de calzas. En ningún caso, aparecen calzas confeccionadas en tejido de seda; todas ellas se confeccionan con lana al exterior, y en el caso del enforro, puede variarse entre lienzo ${ }^{7}$ o lana fina. En su mayoría, como los jubones, se guarnecían con olanda $^{8}$, y en menor cantidad, también con flandes, usados respectivamente en combinación para guarnecer con lienzos de la tierra bastos ${ }^{9}$, probablemente dependiendo de la zona de roce con la carne y la rigidez buscada. Aproximadamente se necesita 1 vara (algo menos de metro ${ }^{10}$ ) de tejido para cada par, dependiendo la cantidad con el tipo de paño, que posiblemente se cortaría en el sentido la urdimbre para evitar la deformación del tejido.

Para el Rey se confeccionan calzas negras $\left(7\right.$ de melinas ${ }^{11}$ y 7 de liria ${ }^{12}$ de velarte mayor), y en grana-morado (19 de roán ${ }^{13}$ y 4 de florentín $\left.{ }^{14}\right)$, siendo las de melinas las que menos cantidad de paño necesitan, aunque es posible que las variaciones en las

7 El lienzo es un tejido de lino o cáñamo, sin tintura, blanqueado, o teñido en colores. Los bastos se utilizan principalmente para forros, valorándose su tiesura; y los finos en prendas delicadas en contacto con la piel como camisones o sábanas, y objetos desde tocados a fruteros. En Castilla se importaron lienzos de Holanda, Flandes y Bretaña, que tomaron el nombre de la región o incluso la ciudad desde donde inicialmente se producían, que en muchos casos acabaron por convertirse en términos para definir un determinado tejido, vid.: DIAGO HERNANDO, Hernando, La industria y el comercio de productos textiles en Europa: siglos XI al XV, Madrid, Arco Libros, 1997. Se diferenciaba aquellos de especial calidad con el término "delgado". También se produjeron lienzos, llamados "de la tierra", que resultaban mucho más económicos y eran de calidad "basta", utilizados para forros.

8 A finales de la Edad Media, "olanda" era el término para designar un tipo de lienzo especialmente delicado, de punto llano, compacto, y color blanco leche. MARTíNEZ MELÉNDEZ, María del Carmen, El nombre de los tejidos en castellano antiguo, Granada, Universidad de Granada, 1989, pp. 490-502.

9 Sería el tejido de lana más común, fabricado en ciudades como Palencia, Segovia o Ávila. En la Cámara se utilizan dos variedades: una común, sin especificar, y otra que se denomina "basto".

10 Las medidas en el texto se ofrecen traspasadas a metros y centímetros, pero en la documentación se utilizan como unidades de medida: la vara castellana (83 centímetros, de acuerdo con la medida grabada probablemente en el siglo XV, y conservada en una columna de la Plaza Chica de Zafra), palmo (24 centímetros) y de dedo ( 2 centímetros).

11 Paño de lana de calidad procedente de Malinas, a veces era transportado sin teñir y terminado de elaborar en Castilla. Cada vara cuesta a 800 maravedís. Al precio de los tejidos de lana de calidad habría que sumar el tundido: igualar con tijeras el pelo de los paños, para un aspecto uniforme y refinado, que se llevaba a cabo en el momento de su registro. Vid.: MARTÍNEZ MELÉNDEZ, María del Carmen (1989), op.cit., pp. $227-230$

12 Originalmente procedente de Lierre, (en castellano Liera), Bélgica. Existen al menos 4 variedades: clusquí, de velarte, de velarte mayor y menor, con precios que oscilan entre 520 y 150 maravedís la vara. Según María del Carmen Martínez Meléndez, era fino y en principio negro, enfurtido y lustroso, cubierto de fina lanilla. Inicialmente se importaría del extranjero (están documentados los procedentes de Malinas) pero también se fabricó en Cuenca. Ibid., pp. 227-230. En la anotación de uno de los testeos se menciona que se trata en realidad de paño de Malinas, y su precio es de 520 maravedís (AGS C y SR, Leg.42 Fol.1 - e) 6 1r (en el margen izquierdo, sobre la línea de escritura): "la vara de la liria que / dize que heran Mellinas a 520. Lo / qual juró Allfonso García que hera Mellinas. / [Rúbrica]".

13 Paños de lana procedentes de Rouen, en la Cámara hay tres variedades: mayor, menor y común. El pardillo cuesta de 380 a 420 maravedís la vara. El rey regala prendas de roán a sus músicos, Cantor de Capilla, Cubijera de Cámara, donceles, Repostero de camas... y nobles como el Arzobispo de Toledo.

14 Se fabricaba en Inglaterra y Segovia, pero cabe pensar que procediese de Florencia y fuese de excepcional calidad, porque cuesta a 830 maravedís la vara. 
cantidades viniesen dadas por la necesidad de tundir algunos de los materiales antes de proceder a la confección.

Todas estas calzas eran lisas y con las dos patas del mismo color ${ }^{15}$, y tenían avampiés, a excepción de las de paño de grana de florentín. El resto de calzas dadas como regalo o librea son pardillas, de roán menor o mayor, sin avampiés, y ya estaban en la Cámara antes del 4 de abril de 1453. Es importante aclarar que no se menciona en ningún caso el término "librea", pero muchas de las prendas, e incluso trajes completos, parecen tener esta finalidad. (fig.1)

\begin{tabular}{|c|c|c|}
\hline \multicolumn{3}{|l|}{ Calzas cortadas para el Rey: } \\
\hline TEJIDO & COLOR & AVAMPIÉS \\
\hline Malinas de velarte mayor ( 7$)$ & negro & si \\
\hline Roán (19) & grana-morado & si \\
\hline Liria de velarte mayor (7) & negro & si \\
\hline Florentín(4) & grana-colorado & no \\
\hline \multicolumn{3}{|l|}{ Otras calzas: } \\
\hline Roán menor & pardillo & no \\
\hline Roán mayor & pardillo & no \\
\hline
\end{tabular}

Fig. 1. Calzas cortadas y dadas en la Cámara de Juan II entre el 4 de abril y el 31 de diciembre de 1453. Fuente: elaboración propia a partir de los datos extraídos del AGS, C y SR, leg. 42, fol. 1.

El calcetero en la Cámara, Alfonso de Cadahalso, confeccionaba las calzas en grupos de hasta 7 pares cortadas del mismo tejido, y en un día podían cortarse hasta 11 pares de diferentes materiales. Con los jubones, las calzas fueron las prendas confeccionadas en mayor número en 1453. Esto puede deberse a que por su uso eran prendas que sufrirían un mayor desgaste que otras, ya que con las interiores son indispensables en el traje diario, además de que al encontrarse más cerca de la piel serían

15 Las calzas rayadas no aparecerán en Castilla hasta finales de siglo, según BERNIS, Carmen (1950), op. cit., p. 200. Las calzas con una pata de cada color serían de uso habitual. Pueden verse en el Retablo de Santa Úrsula del Convento de San Pablo de Palencia, ca.1425-50, (Madrid), Museo Nacional del Prado, P07630. 
más susceptibles a la sudoración. Otra posibilidad, dada la ausencia de camisas y bragas, es que la Cámara organizase la producción cíclica de prendas de acuerdo con su lugar dentro del traje. Para demostrar esta hipótesis sería necesario hallar registros previos, pero sería una explicación a la ausencia de camisas, que son abundantes (y en ocasiones muy ricas) en inventarios realizados a la muerte de otros nobles castellanos contemporáneos.

\section{LOS JUBONES}

El jubón de este momento cubre el torso desde el cuello, con un largo variable: hasta la cintura, o con faldeta bajo los glúteos. Abierto por la parte central del pecho, se cierra al torso con botones o con un encordado. Tiene mangas muy ajustadas, también abotonadas o ceñidas, que pueden llevar un pliegue o recogido con almohadillado a la altura superior del brazo para ensanchar la línea de los hombros, llamado brahón. ${ }^{16}$

En algunas partes está relleno con estofa, que es la principal herramienta para el modelado del torso de acuerdo con la silueta que se ha descrito previamente. La estofa serviría además como acolchado cuando sobre el jubón se vestía coraza. Aunque en este documento no aparece mencionada ninguna coraza de armar, son muchas las representaciones del manuscrito II / 3009 que muestran a reyes armados, en las que sin embargo no es visible el collar del jubón, que queda oculto bajo el gorjal.

El collar (que actualmente podría equipararse a un cuello de tirilla), en este momento es alto ${ }^{17}$, de unos 8 centímetros aproximados de ancho según se deduce de las proporciones de sus representaciones. De consistencia muy rígida, se cierra en sus extremos sobre la nuez con un pequeño cordel destensado, bajo el cual puede verse a veces la camisa.

Con las mangas, el collar es la única parte visible en el conjunto del traje, por lo que a veces se renovarían, o se realizarían en un material diferente, pero esto no sucede en ningún caso entre los jubones confeccionados en la Cámara: el Rey regala varios de sus jubones, y deja el resto, así como sus calzas "viejas", a su Mozo de Cámara ${ }^{18}$ Alfonso de Illescas en un albalá de descargo, sobreentendiéndose que para ser reutilizados de alguna forma, aunque no ya en la Cámara. (fig.2)

Alfonso de Illescas recibe del Comprador de la Cámara, Lope de Villacorta, una pieza de 33 varas y 5 sesmas de setuní raso clemesín, lo que actualmente entenderíamos como raso carmesí, destinada específicamente a jubones para el Rey. El precio

\footnotetext{
16 Plegado a modo de rosca en el hombro. Vid.: BERNIS, Carmen (1979), op. cit., p. 46 . El término no aparece en la documentación consultada.

17 BERNIS, Carmen, «Pedro Berruguete y la moda: algunas aclaraciones cronológicas sobre su obra», en Archivo Español de Arte, XX, 12, 1959, p. 26.

18 Alfonso de Illescas. Era el encargado del almacenaje y transporte de materiales textiles y prendas de la Cámara, así como quien supervisaba su entrada con el pago a mercaderes y recepción de Reposteros y Compradores de Cámara, y la salida de materiales y prendas por tajos y las datas. Una descripción detallada del cargo de mozo de cámara y otros correspondientes a la Cámara de Juan II aparece en CAÑAS GÁLVEZ, Francisco de Paula, "La Cámara de Juan II", en Evolución y estructura de la Casa Real de Castilla, vol. I, Madrid, 2010, pp. 84-195.
} 


\begin{tabular}{|c|c|c|c|}
\hline \multicolumn{3}{|c|}{ Jubones cortados para el Rey: } & \\
\hline TEJDO & COLOR & $\begin{array}{l}\text { Precio total de los materiales por prenda } \\
\text { (incluyendo enforros, guarniciones y estofas) }\end{array}$ & \\
\hline Setuní raso ( 7 jubones) & clemesín & 2222 maravedís & \\
\hline Damasco (6 jubones) & clemesín & & \\
\hline Setuní raso (4 jubones) & verde & 1342 maravedis & \\
\hline Setuní raso (3 jubones) & pardillo & 1382 maravedis & \\
\hline Damasco (5 jubones) & pardillo & 1802 maravedís & \\
\hline \multicolumn{3}{|c|}{ Jubones cortados para los donceles del Rey: } & \\
\hline Damasco (3 jubones) & negro & & \\
\hline \multicolumn{4}{|c|}{ Jubones viejos usados por el Rey que salen de la Cámara: } \\
\hline TEJDO & COLOR & Dado a: & Cargo: \\
\hline Damasco & pardillo & Castilla & Rey de armas \\
\hline Damasco & verde & $\begin{array}{l}\text { Sancha de Salazar } \\
\text { (para su hijo) }\end{array}$ & Cubiiera \\
\hline Damasco & verde & Juan de Juanas & Repostero de camas \\
\hline Damasco & clemesín & Juan de Monsalve & Maestresala \\
\hline
\end{tabular}

Fig. 2. Jubones cortados y dados en la Cámara de Juan II entre el 4 de abril y el 31 de diciembre de 1453. Fuente: elaboración propia a partir de los datos extraídos del AGS, C y SR, leg. 42, fol. 1.

por vara de esta seda sin oro es de 480 maravedís. El mismo tipo de tejido, pero en color verde y con oro, tiene un precio de 260 maravedís por vara, probablemente debido a que aunque contiene oro, el precio de su tintura era más barato, y en tono pardillo, de 270 maravedís.

Otros tejidos con oro que entran en la Cámara destinados a jubones, son el damasco pardillo y el amarillo, ambos a 450 maravedís la vara, y el tapete negro, a 500 . Dentro de las sedas con oro, destacarían por su vistosidad aquellas denominadas "de lauores", como el setuní morado de labores y el azul alcachofado, a 600 y 500 maravedís la vara, respectivamente. 
Los jubones para el Rey y sus pajes se realizan por tanto en tejido exterior de seda (rasos y damascos ${ }^{19}$ negro, pardillo, verde y carmesí), que luego se guarnece y forra con lienzos, al igual que las calzas, y se estofa con lienzo de Bretaña basto ${ }^{20}$.

La peculiaridad de los enforros de los jubones se encuentra en que en cada prenda se combinan diferentes tipos de lienzos: olanda (a 50 maravedís la vara) en una cantidad de 4 varas por prenda, 6 varas de bretaña basta para estofas (a 10 maravedís la vara), y en menor proporción, una vara y media, lienzo de la tierra (a 28 maravedís la vara), o excepcionalmente bretaña. Estas medidas son iguales para todos los jubones confeccionados, e independientes del tejido exterior, ya sea raso o damasco.

Para el exterior del jubón de un hombre adulto se necesitaban 3 varas y una tercia (aproximadamente 2,75 metros) de tejido si se usaba damasco, y 4 varas (unos 3,30 metros) de tejido aproximadamente, si se utilizaba setuní raso ${ }^{21}$, más flexible. El precio de los materiales para cada uno de estos jubones, puede deducirse de las calidades y cantidades de tejido utilizadas, sin embargo no es posible calcular el precio final de la prenda, ya que el sastre Pedro de Matilla ${ }^{22}$ no cobra por la confección de cada una de ellas, sino que "es sastre en la Cámara", aunque no exista el título de sastre de Cámara como tal.

\section{EL TRAJE COORDINADO}

Los tejidos exteriores de los jubones aparecen combinados con varias guarniciones para espadas de traer delante, así como guarniciones para armaduras de cabeza, con los que parece probable que conformasen un mismo traje. Así encontramos en combinación los siguientes jubones y objetos: un jubón de setuní raso morado de labores, una guarnición de espada y el enforro de una armadura de cabeza; jubones de setuní raso clemesín a juego con el enforro de un sombrero de paja blanco; y aunque no llegue a confeccionarse, sí se menciona un tapete negro para jubones, con el que se guarnece otra funda de espada.

19 Es el tejido que aparece en más tonos, pero se desconoce el precio de la mayoría de ellos, porque al encontrarse ya en la Cámara en 1453, aparecen en datas y tajos, pero no en costos.

20 Es probable que su nombre se refiera a un tipo de tejido, más que a su procedencia. Se utiliza en combinación con la olanda. Cuesta 10 maravedís la vara. GARCÍA FERNÁNDEZ, Máximo, DURÁN PUJOL, Montserrat y DÁVILA CORONA, Rosa María, Diccionario Histórico de telas y tejidos castellano-catalán, Salamanca, Junta de Castilla y León, Consejería de Cultura y Turismo 2004, pp. 136-200.

21 El setuní raso ya aparece con el nombre abreviado de "raso" en este documento. AGS. C y SR, leg. 42, fol. $1-\mathrm{c})$. fol. $1 \mathrm{r}$

22 "Se trata de una prenda que gozaba de una buena consideración, tratándose de la más cara [en su confección], existiendo, incluso, un comercio de jubones de segunda mano realizados por los llamados ropavejeros. En este sentido podemos decir que la silueta clásica del siglo XV era la de un hombre con jubón, lo que explica que los hubiese de todas las formas y tamaños, precios y calidades, básicamente se elaboraban con fustán teñido de diversos colores (olmo, acebuche, etc.), o de diversas procedencias o maneras (levante o parella), paño o seda. Había jubones para adultos y para mozos y pajes, según la edad: de 6 a 9 o de 10 a 15 años”. PUÑAL FERNÁNDEZ, Tomás, «El ordenamiento de precios y salarios de Juan II en 1442: estudio histórico-diplomático», en Espacio, Tiempo y Forma: revista de la facultad de Geografia e Historia, Serie 3. Historia Medieval, 14, 2001, p. 291. 
En el primer caso, pese a que como ya se ha mencionado, el collar del jubón no es visible si el caballero va armado, la coordinación entre jubón y enforro de la armadura de cabeza, responde al momento en que el Rey se presentase con esta última quitada. Dentro del protocolo real, existiría incluso una figura encargada de portar esta armadura de cabeza, un paje que portaría esta prenda durante el camino:

“[...] cortó más el dicho Rodrigo armero en la / dicha cámara: vna guarniçión e enforro de setuní de lauores ${ }^{15}$ morado para vn $a$ armadura de cabeza del dicho sennor Rrey, / que trahe el su paje por camino. Que entró en la dicha guar/niçión vna vara e media de la dicha seda. (en el margen derecho) $<1$ vara media setuní $>$ "23.

El trabajo en la Cámara tanto de Pedro de Matilla como de Alfonso de Cadahalso, que van siguiendo al Rey allá donde se desplaza, implica también que en la Cámara no existe el oficio de jubonero, y la confección de estas prendas, que debía de ser bastante compleja, recae en el sastre.

Como sucede con calzas y jubones, el resto de objetos mencionados no son confeccionados por la misma persona, siendo el armero su encargado en el caso de las armas, y el sedero en el del sombrero, de tal manera que aunque en este trabajo se trata sólo de un grupo de prendas en el amplio conjunto de las que conformaban el traje masculino del siglo XV, se demuestra que para un mismo traje era necesaria la intervención de diferentes profesionales, y que éstos trabajarían coordinados en torno a un diseño de traje en común, para el que se creaban prendas o se modificaba alguna de sus partes, con el fin de conjuntarlas usando el mismo tejido.

23 AGS, C y SR, leg.42 fol.1, 1 - c) 2 fol. 1r 